\title{
Pseudoproduction, Pseudocost and Profit Functions in Monopoly from the Dual Perspective
}

\author{
Ilko Vrankić, Mira Krpan
}

Faculty of Economics and Business, University of Zagreb, Croatia

\section{Abstract}

Background: Duality in the microeconomic theory enables us to represent consumers' preferences and production technology with various dual functions satisfying certain regularity conditions. Objectives: Since the basis for the application of duality in the microeconomic theory is the price taking behaviour, this paper takes the challenge of applying principles of duality to a monopolistic case where a single producer has an influence on the price which it charges for its product. Methods/Approach: The standard approach of deriving the profit function for the monopolist from the production function and the defined pseudoproduction function is accompanied by an alternative approach in which the starting point is the pseudocost function. Starting from the derived profit function, the pseudoproduction function and the pseudocost functions are recovered and a version of Hotelling's lemma is given. Results: The structure of the profit maximization problem in a monopolistic case was made similar to the structure of the profit maximization problem in the perfectly competitive case and it is shown that all starting functions can be recovered back from derived functions. A version of Hotelling's lemma is illustrated, which brings us indirectly from the profit function to the supply function. Conclusions: By introducing the pseudoproduction function in the profit maximization model of a monopolist, the structure of the problem becomes similar to the perfectly competitive case and duality results can be applied. The profit function is derived from the pseudoproduction and the pseudocost function, and all starting functions are recovered back from the derived profit function.

Keywords: duality, pseudoproduction function, pseudocost function, profit function, Hotelling's lemma

JEL classification: D

Paper type: Research article

Received: Feb 22, 2016

Accepted: Jul, 28, 2016

Citation: Vrankić, I., Krpan, M. (2016), "Pseudoproduction, Pseudocost and Profit Functions in Monopoly from Dual Perspective", Business Systems Research, Vol. 7, No. 2, pp. 49-64.

DOI: 10.1515/bsrj-2016-0012 


\section{Introduction}

Since the introduction of duality in microeconomic theory and acknowledgments of its advantages from a theoretical and empirical standpoint, many applications followed and are still widespread (Diewert, 1982; Briec, Kerstens, Eeckaut, 2004; Kuosmanen, 2003; Taylor, 1989). Duality in microeconomic theory includes derivation and recovering of the alternative representations of the consumer preferences and the production technology (Blume, 2008; Shepard, 1970).

The basis for the application of duality in microeconomic theory is the price taking assumption, and the question is how can the principles of duality be applied in the case of a monopolistic firm where the single producer has an influence on the price which he charges for the product (Appelbaum, 1975; Diewert, 1982; Lau, 1978).

Goal of the paper is to derive all alternative representations of monopolist's technology and to show how every function can be obtained from the other. To accomplish this, we derive the profit function for the monopolist for the given demand function starting from the primal production function and defined pseudoproduction function and then obtain the same profit function starting from the pseudocost function. Furthermore, starting from the derived profit function, we show how the pseudoproduction function can be recovered. Finally, to give a complete treatment of duality in monopolistic case which represents the scientific contribution of the paper, the pseudocost function is derived from the profit function.

The most important result in duality theory from an empirical standpoint is Hotelling's lemma (Mas - Colell, Whinston, Green, 1995), which enables us to obtain the supply function and the input demand functions by simple differentiation of the profit function satisfying certain regularity conditions. We discuss it in the case of monopolist and illustrate a version of it.

\section{From the production function to the profit function}

A monopolist who faces the following inverse demand function (Diewert, 1982)

$$
p_{0}=w D\left[x_{0}\right] \text {, }
$$

is analysed, where $p_{0}$ is the price of the monopolist's product and $w>0$ represents the influence on demand of "other variables", for example income, and D is a function of $x_{0}$ for which $D^{\prime}\left(x_{0}\right)<0$. Therefore, the demand function a monopolist faces is negatively sloped. Given the demand function, monopolist chooses price and quantity combination to maximise profit. Furthermore, the production function $x_{0}=F(\mathbf{x})$ characterizes the monopolist's technology, where $\mathbf{x}$ is the vector of inputs used in the production of the monopolist's product whose quantity is represented by $x_{0}$.

Our goal is to formulate the profit function as a function of input quantities. The total revenue function is equal to the product of price and quantity. Price is represented as an inverse demand function in (1). So, the total revenue function is described by

$$
p_{0} x_{0}=w D[F(\mathbf{x})] F(\mathbf{x})=w F(\mathbf{x}) D[F(\mathbf{x})]
$$

By subtracting input costs from revenue, the profit maximization problem is represented as 


$$
\max _{\mathbf{x}} w F(\mathbf{x}) D[F(\mathbf{x})]-\mathbf{w} \mathbf{x},
$$

where $\mathbf{w}$ is the vector of input prices. To assure that the formulated model has a solution, it is assumed that sufficient regularity conditions are satisfied (Appelbaum, 1975; Diewert, 1982; Lau, 1978).

Regardless of the market structure in which the producer operates, the first order necessary conditions bring us to the equality between the marginal revenue and marginal cost. In the perfectly competitive case marginal revenue is equal to the product of price, which is a parameter for the firm, and the marginal product which is determined by the firm's technology (Diewert, 1982). In order to represent the first order necessary conditions for the monopolist in the similar way and to apply the know results from duality theory in microeconomics, we form the pseudoproduction function (Diewert, 1982)

$$
F^{*}(\mathbf{x})=F(\mathbf{x}) D[F(\mathbf{x})]=\frac{p_{o} x_{0}}{w} .
$$

It can be interpreted as a deflated revenue function because it is equal to the total revenue divided by the parameter $w$ in the inverse demand function. This parameter $w$ will be interpreted as the price of the pseudo product. Therefore, the first order necessary conditions for the monopolist's profit maximization problem reduce to

$$
w \frac{\partial F^{*}(\mathbf{x})}{\partial x_{i}}=w_{i}, \forall i
$$

The monopolist will hire the levels of inputs for which the marginal revenue of the corresponding input, $w \frac{\partial F^{*}(\mathbf{x})}{\partial x_{i}}$, is equal to its marginal cost, $w_{i}$. We assume that the formulated problem has an interior solution and that the monopolist takes input prices as given.

The next step includes solving the first order necessary conditions and that leads us to the input demand functions, which give us the profit maximizing input quantities. To obtain the optimal product quantity of a monopolist, we substitute the derived input demand functions in the production function and get the monopolist's supply function for the given demand function. Finally, substitution of the derived functions in the goal function for the formulated model gives us the monopolist's profit function for the given demand function.

Below we illustrate the whole model by starting from the production function which characterizes decreasing returns to scale (Mas - Colell, Whinston, Green, 1995; Oraić, 2011),

$$
x_{0}=f\left(x_{1}, x_{2}\right)=x_{1}^{\frac{1}{4}} x_{2}^{\frac{1}{4}} .
$$

Demand behaviour is represented by an inverse linear demand function,

$$
p_{0}=w\left(a-b x_{0}\right) .
$$

Taking into account the chosen functional forms for the production function and the inverse demand function, the pseudoproduction function defined in (4) is represented by the following function

$$
F^{*}(\mathbf{x})=\left(a-b x_{1}^{\frac{1}{4}} x_{2}^{\frac{1}{4}}\right) x_{1}^{\frac{1}{4}} x_{2}^{\frac{1}{4}}=a x_{1}^{\frac{1}{4}} x_{2}^{\frac{1}{4}}-b x_{1}^{\frac{1}{2}} x_{2}^{\frac{1}{2}} .
$$


Therefore, the profit maximization model for the monopolist is

$$
\max _{x_{1} \cdot x_{2}} w\left(a x_{1}^{\frac{1}{4}} x_{2}^{\frac{1}{4}}-b x_{1}^{\frac{1}{2}} x_{2}^{\frac{1}{2}}\right)-w_{1} x_{1}-w_{2} x_{2} .
$$

Differentiation of the goal function with respect to the choice variables brings us to the following system of equations which represent first order necessary conditions,

$$
\begin{aligned}
& w \frac{\partial F^{*}(\mathbf{x})}{\partial x_{1}}=w\left(\frac{a}{4} x_{1}^{-\frac{3}{4}} x_{2}^{\frac{1}{4}}-\frac{b}{2} x_{1}^{-\frac{1}{2}} x_{2}^{\frac{1}{2}}\right)=\frac{w}{4} x_{1}^{-\frac{3}{4}} x_{2}^{\frac{1}{4}}\left(a-2 b x_{1}^{\frac{1}{4}} x_{2}^{\frac{1}{4}}\right)=w_{1} \\
& w \frac{\partial F^{*}(\mathbf{x})}{\partial x_{2}}=w\left(\frac{a}{4} x_{1}^{\frac{1}{4}} x_{2}^{-\frac{3}{4}}-\frac{b}{2} x_{1}^{\frac{1}{2}} x_{2}^{-\frac{1}{2}}\right)=\frac{w}{4} x_{1}^{\frac{1}{4}} x_{2}^{-\frac{3}{4}}\left(a-2 b x_{1}^{\frac{1}{4}} x_{2}^{\frac{1}{4}}\right)=w_{2}
\end{aligned}
$$

Solution to the above system of equations gives input demand functions. Below we analyse the constraints that are implied by the sufficient second order necessary conditions (Lau, 1978). Since the parameter $w$ is positive, requirements for the sufficient conditions are:

$$
\frac{\partial F^{* 2}\left(x_{1}, x_{2}\right)}{\partial x_{1}^{2}}<0
$$

and

$$
\frac{\partial F^{* 2}\left(x_{1}, x_{2}\right)}{\partial x_{1}^{2}} \frac{\partial F^{* 2}\left(x_{1}, x_{2}\right)}{\partial x_{2}^{2}}-\left(\frac{\partial F^{* 2}\left(x_{1}, x_{2}\right)}{\partial x_{1} \partial x_{2}}\right)^{2}>0
$$

From the first order necessary conditions in (10) it follows

$$
\frac{\partial F^{* 2}\left(x_{1}, x_{2}\right)}{\partial x_{1}^{2}}=-\frac{3 a}{16} x_{1}^{-\frac{7}{4}} x_{2}^{\frac{1}{4}}+\frac{b}{4} x_{1}^{-\frac{3}{2}} x_{2}^{\frac{1}{2}}=-\frac{3}{16} x_{1}^{-\frac{7}{4}} x_{2}^{\frac{1}{4}}\left(a-\frac{4 b}{3} x_{1}^{\frac{1}{4}} x_{2}^{\frac{1}{4}}\right)<0 \text {. }
$$

Since

$$
\begin{gathered}
\frac{\partial F^{* 2}\left(x_{1}, x_{2}\right)}{\partial x_{1}^{2}}=-\frac{3 a}{16} x_{1}^{-\frac{7}{4}} x_{2}^{\frac{1}{4}}+\frac{b}{4} x_{1}^{-\frac{3}{2}} x_{2}^{\frac{1}{2}}=-\frac{1}{16} x_{1}^{-\frac{7}{4}} x_{2}^{\frac{1}{4}}\left(3 a-4 b x_{1}^{\frac{1}{4}} x_{2}^{\frac{1}{4}}\right)<0 \\
\frac{\partial F^{* 2}\left(x_{1}, x_{2}\right)}{\partial x_{2}^{2}}=-\frac{1}{16} x_{1}^{\frac{1}{4}} x_{2}^{-\frac{7}{4}}\left(3 a-4 b x_{1}^{\frac{1}{4}} x_{2}^{\frac{1}{4}}\right) \\
\frac{\partial F^{* 2}\left(x_{1}, x_{2}\right)}{\partial x_{1}^{2}} \frac{\partial F^{* 2}\left(x_{1}, x_{2}\right)}{\partial x_{2}^{2}}=\frac{1}{256} x_{1}^{-\frac{3}{2}} x_{1}^{-\frac{3}{2}}\left(3 a-4 b x_{1}^{\frac{1}{4}} x_{2}^{\frac{1}{4}}\right)^{2}
\end{gathered}
$$




$$
\begin{gathered}
\frac{\partial F^{* 2}\left(x_{1}, x_{2}\right)}{\partial x_{2} \partial x_{2}}=\frac{1}{16} x_{1}^{-\frac{3}{4}} x_{2}^{-\frac{3}{4}}\left(a-4 b x_{1}^{\frac{1}{4}} x_{2}^{\frac{1}{4}}\right) \\
\left(\frac{\partial F^{* 2}\left(x_{1}, x_{2}\right)}{\partial x_{2} \partial x_{2}}\right)^{2}=\frac{1}{256} x_{1}^{-\frac{3}{2}} x_{2}^{-\frac{3}{2}}\left(a-4 b x_{1}^{\frac{1}{4}} x_{2}^{\frac{1}{4}}\right)^{2} \\
\frac{\partial F^{* 2}\left(x_{1}, x_{2}\right)}{\partial x_{1}^{2}} \frac{\partial F^{* 2}\left(x_{1}, x_{2}\right)}{\partial x_{2}^{2}}-\left(\frac{\partial F^{* 2}\left(x_{1}, x_{2}\right)}{\partial x_{1} \partial x_{2}}\right)^{2}=\frac{1}{256} x_{1}^{-\frac{3}{2}} x_{1}^{-\frac{3}{2}}\left(3 a-4 b x_{1}^{\frac{1}{4}} x_{2}^{\frac{1}{4}}\right)^{2}-\frac{1}{256} x_{1}^{-\frac{3}{2}} x_{2}^{-\frac{3}{2}}\left(a-4 b x_{1}^{\frac{1}{4}} x_{2}^{\frac{1}{4}}\right)^{2} \\
\frac{\partial F^{* 2}\left(x_{1}, x_{2}\right)}{\partial x_{1}^{2}} \frac{\partial F^{* 2}\left(x_{1}, x_{2}\right)}{\partial x_{2}^{2}}-\left(\frac{\partial F^{* 2}\left(x_{1}, x_{2}\right)}{\partial x_{1} \partial x_{2}}\right)^{2}=\frac{1}{256} x_{1}^{-\frac{3}{2}} x_{1}^{-\frac{3}{2}}\left[\left(3 a-4 b x_{1}^{\frac{1}{4}} x_{2}^{\frac{1}{4}}\right)^{2}-\left(a-4 b x_{1}^{\frac{1}{4}} x_{2}^{\frac{1}{4}}\right)^{2}\right]>0 .
\end{gathered}
$$

Dividing both expressions in (10) gets us to

$$
\frac{x_{2}}{x_{1}}=\frac{w_{1}}{w_{2}} .
$$

In other words, the necessary condition for the profit maximization problem implies that the marginal rate of technical substitution, which is equal to the ratio of marginal products of inputs, $M R T S=\frac{\partial f(L, K) / \partial x_{1}}{\partial f(L, K) / \partial x_{2}}=\frac{x_{2}}{x_{1}}$, is equal to the ratio of input prices, $\frac{w_{1}}{w_{2}}$

If we express $x_{2}$ as a function of $x_{1}$, we get

$$
x_{2}=\frac{w_{1}}{w_{2}} x_{1}
$$

By substituting it in one of (10), we get the following equation

$$
\frac{w}{4} x_{1}^{-\frac{3}{4}} \frac{w_{1}^{\frac{1}{4}}}{w_{2}^{\frac{1}{4}}} x_{1}^{\frac{1}{4}}\left(a-2 b x_{1}^{\frac{1}{2}} \frac{w_{1}^{\frac{1}{4}}}{w_{2}^{\frac{1}{4}}}\right)=w_{1}
$$

whose solution gives the monopolist's demand function for the first input,

$$
x_{1}^{*}=\frac{a^{2} w^{2} w_{2}^{\frac{1}{4}}}{4 w_{1}^{\frac{1}{2}}\left(2 w_{1}^{\frac{1}{2}} w_{2}^{\frac{1}{2}}+b w\right)^{2}} .
$$

By inserting it in (22), we get the monopolist's demand function for the second input, 


$$
x_{2}^{*}=\frac{a^{2} w^{2} w_{1}^{\frac{1}{4}}}{4 w_{2}^{\frac{1}{2}}\left(2 w_{1}^{\frac{1}{2}} w_{2}^{\frac{1}{2}}+b w\right)^{2}}
$$

Getting back to our production function in (6), and inserting the derived input demand functions in it, we get the supply function for the monopolist, for the given demand function,

$$
x_{0}^{*}=\frac{a w}{2\left(2 w_{1}^{\frac{1}{2}} w_{2}^{\frac{1}{2}}+b w\right)} \text {. }
$$

Finally, substitution of derived supply and demand functions in the goal function of a monopolist gets us to the profit function

$$
\begin{aligned}
& \pi^{*}=w\left[\frac{a^{2} w}{2\left(2 w_{1}^{\frac{1}{2}} w_{2}^{\frac{1}{2}}+b w\right)}-\frac{a^{2} w^{2} b}{4\left(2 w_{1}^{\frac{1}{2}} w_{2}^{\frac{1}{2}}+b w\right)^{2}}\right]-\frac{a^{2} w^{2} w_{1}^{\frac{1}{2}} w_{2}^{\frac{1}{2}}}{4\left(2 w_{1}^{\frac{1}{2}} w_{2}^{\frac{1}{2}}+b w\right)^{2}}-\frac{a^{2} w^{2} w_{1}^{\frac{1}{2}} w_{2}^{\frac{1}{2}}}{4\left(2 w_{1}^{\frac{1}{2}} w_{2}^{\frac{1}{2}}+b w\right)^{2}} \\
& \pi^{*}=\frac{a^{2} w^{2}}{2\left(2 w_{1}^{\frac{1}{2}} w_{2}^{\frac{1}{2}}+b w\right)}-\frac{a^{2}}{4\left(2 w_{1}^{\frac{1}{2}} w_{2}^{\frac{1}{2}}+b w\right)^{2}}\left(2 w_{1}^{\frac{1}{2}} w_{2}^{\frac{1}{2}}+b w\right) \\
& \pi^{*}=\frac{a^{2} w^{2}}{4\left(2 w_{1}^{\frac{1}{2}} w_{2}^{\frac{1}{2}}+b w\right)} .
\end{aligned}
$$

\section{From the pseudocost function to the profit function}

Our first approach in deriving the supply function and the profit function for the monopolist started from the production function. This approach can be called the primal approach since the technology is described by primal variables. An alternative method for obtaining the monopolist's profit function for the given demand function is to start from the cost function, $c\left(x_{0}, \mathbf{w}\right)$ (Shepard, 1970). This approach of deriving the monopolist's profit function can be formulated in the following way,

$$
\max _{x_{0}} p_{0} x_{0}-c\left(x_{0}, \mathbf{w}\right)=\max _{x_{0}} w D\left(x_{0}\right) x_{0}-c\left(x_{0}, \mathbf{w}\right) .
$$

The choice variable is the quantity of production. To be able to represent this dual approach as in the perfectly competitive case, the quantity of production, $x_{0}$, can be expressed as the function of pseudoproduction, $y, x_{0}=g(y)$. Consequently, the profit maximization problem reduces to 


$$
\begin{aligned}
& \max _{y} w y-c[\mathbf{w}, g(y)] \\
& =\max _{y} w y-c^{*}(y, \mathbf{w}) .
\end{aligned}
$$

The standard cost function is now represented as a function of the pseudo product and can be called the pseudocost function, $c^{*}(y, \mathbf{w})$.

Our goal is to solve the model and get the monopolist's supply and profit functions. By differentiating the goal function with respect to the pseudo product, we get the first order necessary condition for the profit maximization problem. It includes the following equation which needs to be solved to get us to the pseudoproduction function:

$$
w=\frac{\partial c^{*}(y, \mathbf{w})}{\partial y}
$$

On the left hand side of the upper equation is the parameter $w$ which we interpreted as the price of the pseudo product. In equilibrium it has to be equal to the derivative of the pseudocost function with respect to the pseudo product. Therefore, profit maximization for the given demand function in this model is characterized by the equality between the price of the pseudo product and the marginal pseudocost.

Below we give an illustration of this alternative approach for chosen production function in (6). The cost function as a function of production level is the result of the model of cost minimization for the given level of production. More on derivation and the properties of the cost function can be found in (Mas-Collel, Whinston, Green, 1995).

$$
\begin{aligned}
& c\left(x_{0}, \mathbf{w}\right)=\min _{\mathbf{x}} \mathbf{w x} \\
& \text { subject to } f\left(x_{1}, x_{2}\right)=x_{0} .
\end{aligned}
$$

In our example it is the result of the following optimization model

$$
\begin{aligned}
& c\left(x_{0}, \mathbf{w}\right)=\min _{\mathbf{x}} \mathbf{w} \mathbf{x} \\
& \text { subject to } x_{1}^{\frac{1}{4}} x_{2}^{\frac{1}{4}}=x_{0 .} .
\end{aligned}
$$

First order necessary conditions imply economic efficiency characterized by the equality between the already mentioned marginal rate of technical substitution and the input price ratio $\frac{w_{1}}{w_{2}}$. This confirms that the cost minimization is the necessary condition for the profit maximization. In our case the equation describing economic efficiency is

$$
\operatorname{MRTS}=\frac{x_{2}}{x_{1}}=\frac{w_{1}}{w_{2}}
$$


Expressing the quantity of the second input as a function of the quantity of the first input brings us to

$$
x_{2}=\frac{w_{1} x_{1}}{w_{2}}
$$

The economic interpretation of this curve is that it describes the optimal combinations of inputs at each output level as output expands and is called the long-run expansion path. By inserting it in the constraint of the model we get the conditional demand functions for inputs, $x_{1}\left(w_{1}, w_{2}, x_{0}\right)$ and $x_{2}\left(w_{1}, w_{2}, x_{0}\right)$. They give us the cost minimizing input levels for the given output level,

$$
x_{1}\left(w_{1}, w_{2}, x_{0}\right)=w_{1}^{-\frac{1}{2}} w_{2}^{\frac{1}{2}} x_{0}^{2}
$$

and

$$
x_{2}\left(w_{1}, w_{2}, x_{0}\right)=w_{1}^{\frac{1}{2}} w_{2}^{-\frac{1}{2}} x_{0}^{2} .
$$

Substitution of input demand function in the goal function gives the cost function

$$
c\left(w_{1}, w_{2}, x_{0}\right)=2 w_{1}^{\frac{1}{2}} w_{2}^{\frac{1}{2}}\left(x_{0}\right)^{2} .
$$

Our goal is to express the cost function in terms of the pseudoproduction function. The first step includes relating the pseudoproduction function and the quantity of production from the definition of the pseudoproduction function and our chosen inverse demand function. The following equation follows:

$$
y=x_{0}\left(a-b x_{0}\right)=a x_{0}-b x_{0}^{2} .
$$

Therefore, they are related by the following quadratic equation

$$
b\left(x_{0}\right)^{2}-a x_{0}+y=0,
$$

from which we express the quantity of production as a function of the pseudo product,

$$
x_{0}=\frac{a \pm \sqrt{a^{2}-4 b y}}{2 b} .
$$

The next step includes expressing the cost function as a function of the pseudo product. The way to do this is to insert the previously derived result in the cost function which leads to

$$
c^{*}\left(y, w_{1}, w_{2}\right)=2 w_{1}^{\frac{1}{2}} w_{2}^{\frac{1}{2}}\left(\frac{a \pm \sqrt{a^{2}-4 b y}}{2 b}\right)^{2} .
$$

Therefore, our alternative approach of profit maximization formulated in (29) now becomes 


$$
\max _{y} w y-2 w_{1}^{\frac{1}{2}} w_{2}^{\frac{1}{2}}\left(\frac{a \pm \sqrt{a^{2}-4 b y}}{2 b}\right)^{2} .
$$

By differentiating the goal function with respect to $y$ we get the first order necessary condition which needs to be solved to get us to the pseudoproduction function

$$
\begin{gathered}
\frac{\partial c}{\partial y}= \pm \frac{2 w_{1}^{\frac{1}{2}} w_{2}^{\frac{1}{2}} a}{b \sqrt{a^{2}-4 b y}}-\frac{2 w_{1}^{\frac{1}{2}} w_{2}^{\frac{1}{2}}}{b}=w . \\
w+\frac{2 w_{1}^{\frac{1}{2}} w_{2}^{\frac{1}{2}}}{b}= \pm \frac{2 w_{1}^{\frac{1}{2}} w_{2}^{\frac{1}{2}} a}{b \sqrt{a^{2}-4 b y}} \\
\frac{w b+2 w_{1}^{\frac{1}{2}} w_{2}^{\frac{1}{2}}}{b}= \pm \frac{2 w_{1}^{\frac{1}{2}} w_{2}^{\frac{1}{2}} a}{b \sqrt{a^{2}-4 b y}} \\
\frac{\left.w b+2 w_{1}^{\frac{1}{2}} w_{2}^{\frac{1}{2}}\right)^{2}}{b^{2}}=\frac{4 w_{1} w_{2} a^{2}}{b^{2}\left(a^{2}-4 b y\right)} \\
a^{2}-4 b y=\frac{4 w_{1} w_{2} a^{2}}{\left(w b+2 w_{1}^{\frac{1}{2}} w_{2}^{\frac{1}{2}}\right)^{2}} \\
4 b y=a^{2}-\frac{4 w_{1} w_{2} a^{2}}{\left(w b+2 w_{1}^{\frac{1}{2}} w_{2}^{\frac{1}{2}}\right)^{2}}
\end{gathered}
$$

$y=\frac{a^{2}}{4 b}-\frac{w_{1} w_{2} a^{2}}{b\left(w b+2 w_{1}^{\frac{1}{2}} w_{2}^{\frac{1}{2}}\right)^{2}}=\frac{a^{2}\left(w b+2 w_{1}^{\frac{1}{2}} w_{2}^{\frac{1}{2}}\right)^{2}-4 w_{1} w_{2} a^{2}}{4 b\left(w b+2 w_{1}^{\frac{1}{2}} w_{2}^{\frac{1}{2}}\right)^{2}}=\frac{a^{2}\left[\left(w b+2 w_{1}^{\frac{1}{2}} w_{2}^{\frac{1}{2}}\right)^{2}-4 w_{1} w_{2}\right]}{4 b\left(w b+2 w_{1}^{\frac{1}{2}} w_{2}^{\frac{1}{2}}\right)^{2}}$

$$
y=\frac{a^{2}\left(w^{2} b^{2}+4 w b w_{1}^{\frac{1}{2}} w_{2}^{\frac{1}{2}}+4 w_{1} w_{2}-4 w_{1} w_{2}\right)}{4 b\left(w b+2 w_{1}^{\frac{1}{2}} w_{2}^{\frac{1}{2}}\right)^{2}}=\frac{a^{2} b\left(b w^{2}+4 w w_{1}^{\frac{1}{2}} w_{2}^{\frac{1}{2}}\right)}{4 b\left(w b+2 w_{1}^{\frac{1}{2}} w_{2}^{\frac{1}{2}}\right)^{2}}=\frac{a^{2}}{4} \frac{\left(4 w w_{1}^{\frac{1}{2}} w_{2}^{\frac{1}{2}}+b w^{2}\right)}{\left(2 w_{1}^{\frac{1}{2}} w_{2}^{\frac{1}{2}}+w b\right)^{2}}
$$

The solution of the equation is the pseudoproduction function, 


$$
y=\frac{a^{2}}{4} \frac{4 w w_{1}^{\frac{1}{2}} w_{2}^{\frac{1}{2}}+b w^{2}}{\left(2 w_{1}^{\frac{1}{2}} w_{2}^{\frac{1}{2}}+b w\right)^{2}}
$$

whose substitution in the goal function leads to the same profit function as in the primal approach in (27),

$$
\pi^{*}=w \frac{a^{2}}{4} \frac{4 w w_{1}^{\frac{1}{2}} w_{2}^{\frac{1}{2}}+b w^{2}}{\left(2 w_{1}^{\frac{1}{2}} w_{2}^{\frac{1}{2}}+b w\right)^{2}}-2 w_{1}^{\frac{1}{2}} w_{2}^{\frac{1}{2}} \frac{a^{2} w^{2}}{4\left(2 w_{1}^{\frac{1}{2}} w_{2}^{\frac{1}{2}}+b w\right)^{2}}=\frac{a^{2} w^{2}}{4\left(2 w_{1}^{\frac{1}{2}} w_{2}^{\frac{1}{2}}+b w\right)} .
$$

\section{Hotelling's lemma}

According to Hotelling's lemma (Appelbaum, 1975, 1979; Diewert, 1982; Mas-Collel, Whinston, Green, 1995) differentiating the profit function of a perfectly competitive, price-taking firm with respect to the product price of a firm gives firm's quantity supplied. Similarly, the derivative of the profit function for a monopolist with respect to the price of the factors of production is equal to the firm's optimal quantities of production factors. Since the monopolist is not the price taker, the question is how can be Hotelling's lemma applied in this case.

Our model assumed that the monopolist behaves competitively in the input market and takes input prices as given. Consequently, the derivation of input demand function from the profit function looks like in the perfectly competitive case and it reduces to differentiation of the profit function with respect to the input prices,

$$
-\frac{\partial \pi^{*}}{\partial w_{1}}=\frac{a^{2} w^{2}}{4\left(2 w_{1}^{\frac{1}{2}} w_{2}^{\frac{1}{2}}+b w\right)^{2}} w_{1}^{-\frac{1}{2}} w_{2}^{\frac{1}{2}}=x_{1}^{*}
$$

and

$$
-\frac{\partial \pi^{*}}{\partial w_{2}}=\frac{a^{2} w^{2}}{4\left(2 w_{1}^{\frac{1}{2}} w_{2}^{\frac{1}{2}}+b w\right)^{2}} w_{1}^{\frac{1}{2}} w_{2}^{-\frac{1}{2}}=x_{2}^{*} .
$$

This procedure is not applicable in deriving the supply function of a monopolist for the given demand function since the monopolist is not the price taker. [8] But looking at the profit maximizing model in (3), the product price can be replaced with the parameter $w$, and the production function can be replaced with the pseudoproduction function. In this case differentiation of the profit function with respect to $w$ gives the pseudoproduction function,

$$
y=\frac{\partial \pi^{*}}{\partial w}=\frac{a^{2}}{4} \frac{2 w\left(2 w_{1}^{\frac{1}{2}} w_{2}^{\frac{1}{2}}+b w\right)-b w^{2}}{\left(2 w_{1}^{\frac{1}{2}} w_{2}^{\frac{1}{2}}+b w\right)^{2}}=\frac{a^{2}}{4} \frac{4 w w_{1}^{\frac{1}{2}} w_{2}^{\frac{1}{2}}+b w^{2}}{\left(2 w_{1}^{\frac{1}{2}} w_{2}^{\frac{1}{2}}+b w\right)^{2}} .
$$


Returning back to the definition of the pseudoproduction function from (4) and (7) the supply function for the given demand function can be obtained by solving the following quadratic equation

$$
\begin{aligned}
& b\left(x_{0}^{*}\right)^{2}-a x_{0}^{*}+\frac{\partial \pi^{*}}{\partial w}=0 . \\
& x_{0}^{*}=\frac{a \pm \sqrt{a^{2}-4 b \frac{\partial \pi^{*}}{\partial w}}}{2 b}=\frac{a \pm \sqrt{a^{2}-a^{2} b \frac{4 w w_{1}^{\frac{1}{2}} w_{2}^{\frac{1}{2}}+b w^{2}}{\left(2 w_{1}^{\frac{1}{2}} w_{2}^{\frac{1}{2}}+b w\right)^{2}}}}{2 b} \\
& x_{0}^{*}=\frac{a \pm \sqrt{\frac{a^{2}}{\left(2 w_{1}^{\frac{1}{2}} w_{2}^{\frac{1}{2}}+b w\right)^{2}}\left[\left(2 w_{1}^{\frac{1}{2}} w_{2}^{\frac{1}{2}}+b w\right)^{2}-b\left(4 w w_{1}^{\frac{1}{2}} w_{2}^{\frac{1}{2}}+b w^{2}\right)\right]}}{2 b} \\
& x_{0}^{*}=\frac{a \pm \sqrt{\frac{a^{2}}{\left(2 w_{1}^{\frac{1}{2}} w_{2}^{\frac{1}{2}}+b w\right)^{2}}\left(4 w_{1} w_{2}+b^{2} w^{2}+4 b w w_{1}^{\frac{1}{2}} w_{2}^{\frac{1}{2}}-4 b w w_{1}^{\frac{1}{2}} w_{2}^{\frac{1}{2}}-b^{2} w^{2}\right)}}{2 b} \\
& x_{0}^{*}=\frac{a \pm \sqrt{\frac{4 w_{1} w_{2} a^{2}}{\left(2 w_{1}^{\frac{1}{2}} w_{2}^{\frac{1}{2}}+b w\right)^{2}}}}{2 b}=\frac{a \pm \frac{2 a w_{1}^{\frac{1}{2}} w_{2}^{\frac{1}{2}}}{2 w_{1}^{\frac{1}{2}} w_{2}^{\frac{1}{2}}+b w}}{2 b}=\frac{2 a w_{1}^{\frac{1}{2}} w_{2}^{\frac{1}{2}}+a b w \pm 2 a w_{1}^{\frac{1}{2}} w_{2}^{\frac{1}{2}}}{2 b\left(2 w_{1}^{\frac{1}{2}} w_{2}^{\frac{1}{2}}+b w\right)} .
\end{aligned}
$$

This is the same supply function we' ve already obtained in (26),

$$
x_{0}^{*}=\frac{a w}{2\left(2 w_{1}^{\frac{1}{2}} w_{2}^{\frac{1}{2}}+b w\right)}
$$

\section{From the profit function to the pseudoproduction function}

Until now we showed how the profit function for the monopolist can be derived by starting from the production and the cost functions. Our goal is to start from the derived profit function and to recover both the pseudoproduction function and the cost function. In this section the pseudoproduction function will be recovered. Definition of the profit function as the maximum profit for every combination of input prices, $w_{1}$ and $w_{2}$, and the parameter $w$, brings us to the following inequality

$$
\pi\left(w, w_{1}, w_{2}\right) \geq w F(\mathbf{x}) D[F(\mathbf{x})]-w_{1} x_{1}-w_{2} x_{2} \quad \forall w_{1}, w_{2}, w .
$$

Definition of the pseudoproduction function enables us to rewrite the previous inequality as 


$$
\pi\left(w, w_{1}, w_{2}\right) \geq w F^{*}(\mathbf{x})-w_{1} x_{1}-w_{2} x_{2} \quad \forall w_{1}, w_{2}, w,
$$

from which it follows that the pseudoproduction function is the result of the following optimization problem

$$
F^{*}(\mathbf{x})=\max \left\{y: w y \leq w_{1} x_{1}+w_{2} x_{2}+\pi\left(w, w_{1}, w_{2}\right)\right\}
$$

By normalizing the price of the pseudo product and dividing all the input prices by $w$, $W_{1}=\frac{w_{1}}{w}, W_{2}=\frac{w_{2}}{w}$, the previous optimization problem reduces to

$$
F^{*}(\mathbf{x})=\max \left\{y: y \leq W_{1} x_{1}+W_{2} x_{2}+\pi\left(1, W_{1}, W_{2}\right)\right\}, \forall W_{1}, W_{2}
$$

and

$$
F^{*}(\mathbf{x})=\min _{W_{1}, W_{2}} W_{1} x_{1}+W_{2} x_{2}+\pi\left(1, W_{1}, W_{2}\right)
$$

Its solution is the monopolist's pseudoproduction function.

Below we illustrate how to recover the pseudoproduction function from the profit function. Starting from our derived profit function in (27), the normalized profit function' 1 is $\pi\left(1, W_{1}, W_{2}\right)=\frac{a^{2}}{\frac{1}{2}{ }^{\frac{1}{2}}}$ and the pseudoproduction function can be $4\left(2 W_{1}^{\overline{2}} W_{2}^{\overline{2}}+b\right)$

obtained as the solution to the following optimization problem

$$
F^{*}(\mathbf{x})=\min _{W_{1}, W_{2}} W_{1} x_{1}+W_{2} x_{2}+\frac{a^{2}}{4\left(2 W_{1}^{\frac{1}{2}} W_{2}^{\frac{1}{2}}+b\right)} .
$$

The system of equations that expresses the first order necessary conditions follows,

$$
\begin{aligned}
& \frac{\partial f}{\partial W_{1}}=x_{1}-\frac{a^{2}}{4}\left(2 W_{1}^{\frac{1}{2}} W_{2}^{\frac{1}{2}}+b\right)^{-2} W_{1}^{-\frac{1}{2}} W_{2}^{\frac{1}{2}}=0 \\
& \frac{\partial f}{\partial w_{2}}=x_{2}-\frac{a^{2}}{4}\left(2 W_{1}^{\frac{1}{2}} W_{2}^{\frac{1}{2}}+b\right)^{-2} W_{1}^{\frac{1}{2}} W_{2}^{-\frac{1}{2}}=0
\end{aligned}
$$

\footnotetext{
${ }^{1}$ The profit function $\pi\left(1, \frac{w_{1}}{w}, \frac{w_{2}}{w}\right)$ is actually the conjugate function to the pseudoproduction function $F^{*}(\mathbf{x})$ if $F^{*}(\mathbf{x})$ is concave (Diewert, 1981). More on the conjugacy approach to duality theory can be found in (Lau, 1978).
} 
Multiplying quantities of inputs gives $x_{1} x_{2}=\frac{a^{2}}{16}\left(2 W_{1}^{\frac{1}{2}} W_{2}^{\frac{1}{2}}+b\right)^{-4}$ which implies $2 W_{1}^{\frac{1}{2}} W_{2}^{\frac{1}{2}}+b=\frac{a}{2} x_{1}^{-\frac{1}{4}} x_{1}^{-\frac{1}{4}}$ and $W_{1}^{\frac{1}{2}} W_{2}^{\frac{1}{2}}=\frac{a}{4} x_{1}^{-\frac{1}{4}} x_{1}^{-\frac{1}{4}}-\frac{b}{2}$. The product of the corresponding input with its price reduces to $W_{1} x_{1}=\frac{a}{4} x_{1}^{\frac{1}{4}} x_{2}^{\frac{1}{4}}-\frac{b}{2} x_{1}^{\frac{1}{2}} x_{2}^{\frac{1}{2}}$ and $W_{2} x_{2}=\frac{a}{4} x_{1}^{\frac{1}{4}} x_{2}^{\frac{1}{4}}-\frac{b}{2} x_{1}^{\frac{1}{2}} x_{2}^{\frac{1}{2}}$. Inserting the given results in the goal function gives

$$
F^{*}(\mathbf{x})=2 \frac{a}{4} x_{1}^{\frac{1}{4}} x_{2}^{\frac{1}{4}}-2 \frac{b}{2} x_{1}^{\frac{1}{2}} x_{2}^{\frac{1}{2}}+\frac{a}{2} x_{1}^{\frac{1}{4}} x_{2}^{\frac{1}{4}}=a x_{1}^{\frac{1}{4}} x_{2}^{\frac{1}{4}}-b x_{1}^{\frac{1}{2}} x_{2}^{\frac{1}{2}}
$$

which is the pseudoproduction function we started from.

\section{From the profit function to the pseudocost function}

To give a complete treatment of the duality relationships between various functions describing the monopolist's behaviour, the pseudocost function must be derived starting from the profit function of a monopolist. Given the arbitrarily chosen price of the pseudo product $w^{0}$, the solution to the problem of profit maximization for the given demand function when starting from the pseudocost function of a monopolist was pseudo product $y^{0}$ and the maximum profit was $\pi\left(w^{0}\right)$. To derive the optimization problem that gives the pseudocost function starting from the pseudo profit function, the following experiment will be made. All the other prices of the factors of production will be fixed and the price of the pseudo product is allowed to change. If the price of the pseudo product changes, we can look at the profit of the active and passive monopolist. Passive monopolist stays at the fixed pseudo product $y^{0}$ and moves along the line

$$
\pi=w y^{0}-c\left(y^{0}\right)
$$

For the active monopolist pseudo product changes at every pseudo product price $w$ and consequently he realizes higher profit. Therefore, the following inequality holds

$$
\pi(w) \geq w y^{0}-c\left(y^{0}\right)
$$

which implies

$$
c\left(y^{0}\right) \geq w y^{0}-\pi(w) .
$$

When the price of the pseudo product is equal to $w^{0}$, the previous inequality becomes equality. It follows that the pseudocost function is the result of the following optimization problem

$$
c(y)=\max _{w} w y^{0}-\pi(w)
$$

This is the problem of maximizing the difference between revenue and profit where the choice variable is $w$. The first order necessary condition expresses the equality between the given pseudo product and marginal pseudo profit and confirms a version of Hotelling's lemma, 
(69)

$$
y^{0}=\frac{\partial \pi}{\partial w}
$$

For the chosen production function in our example this first order necessary condition is

$$
y^{0}=\frac{a^{2}}{4} \frac{4 w w_{1}^{\frac{1}{2}} w_{2}^{\frac{1}{2}}+b w^{2}}{\left(2 w_{1}^{\frac{1}{2}} w_{2}^{\frac{1}{2}}+b w\right)^{2}} .
$$

Solving the upper equation gives us the price of the pseudoproduct. But looking at it, it can be concluded that the solution $w$ is already solved in the alternative optimization problem in which the choice variable was the pseudoproduction function and in which we started from the pseudocost function and derived the profit function. This confirms the known result from the duality theory where the solution to the primal optimization problem is submarginal value of the dual function. The procedure is given below.

$$
\begin{aligned}
& y=\frac{a^{2}}{4} \frac{2 w}{\left(2 w_{1}^{\frac{1}{2}} w_{2}^{\frac{1}{2}}+b w\right)}-\frac{b w^{2}}{\left(2 w_{1}^{\frac{1}{2}} w_{2}^{\frac{1}{2}}+b w\right)^{2}} \\
& 4 a^{2} w w_{1}^{\frac{1}{2}} w_{2}^{\frac{1}{2}}+a^{2} b w^{2}=4 y\left(4 w_{1} w_{2}+4 b w w_{1}^{\frac{1}{2}} w_{2}^{\frac{1}{2}}+b^{2} w^{2}\right) \\
& \frac{a^{2} w w_{1}^{\frac{1}{2}} w_{2}^{\frac{1}{2}}}{y}+\frac{a^{2} b w^{2}}{4 y}=4 w_{1} w_{2}+4 b w w_{1}^{\frac{1}{2}} w_{2}^{\frac{1}{2}}+b^{2} w^{2} \\
& \left(\frac{a^{2} b}{4 y}-b^{2}\right) w^{2}+\left(\frac{a^{2} w_{1}^{\frac{1}{2}} w_{2}^{\frac{1}{2}}}{y}-4 b w_{1}^{\frac{1}{2}} w_{2}^{\frac{1}{2}}\right) w-4 w_{1} w_{2}=0 .
\end{aligned}
$$

$$
\begin{aligned}
& w=\frac{\frac{4 b w_{1}^{\frac{1}{2}} w_{2}^{\frac{1}{2}} y-a^{2} w_{1}^{\frac{1}{2}} w_{2}^{\frac{1}{2}}}{y}}{\frac{b\left(a^{2}-4 b y\right)}{2 y}} \pm \frac{\sqrt{\frac{a^{2} w_{1} w_{2}}{y^{2}}-\frac{8 a^{2} b w_{1} w_{2}}{y}+16 b^{2} w_{1} w_{2}+\frac{4 w_{1} w_{2} a^{2} b}{y}-16 b^{2} w_{1} w_{2}}}{\frac{b\left(a^{2}-4 b y\right)}{2 y}} \\
& w=\frac{\frac{-w_{1}^{\frac{1}{2}} w_{2}^{\frac{1}{2}}\left(a^{2}-4 b y\right)}{1}}{\frac{b\left(a^{2}-4 b y\right)}{2}} \pm \frac{\sqrt{\frac{a^{4} w_{1} w_{2}-4 a^{2} b w_{1} w_{2} y}{y^{2}}}}{\frac{b\left(a^{2}-4 b y\right)}{2 y}}
\end{aligned}
$$




$$
\begin{aligned}
w=-\frac{2 w_{1}^{\frac{1}{2}} w_{2}^{\frac{1}{2}}}{b} \pm \frac{\sqrt{\frac{a^{2} w_{1} w_{2}\left(a^{2}-4 b y\right)}{y^{2}}}}{\frac{b\left(a^{2}-4 b y\right)}{2 y}} & =-\frac{2 w_{1}^{\frac{1}{2}} w_{2}^{\frac{1}{2}}}{b} \pm \frac{\frac{a w_{1}^{\frac{1}{2}} w_{2}^{\frac{1}{2}}}{y} \sqrt{a^{2}-4 b y}}{\frac{b\left(a^{2}-4 b y\right)}{2 y}} \\
w & =-\frac{2 w_{1}^{\frac{1}{2}} w_{2}^{\frac{1}{2}}}{b} \pm \frac{2 a w_{1}^{\frac{1}{2}} w_{2}^{\frac{1}{2}}}{b \sqrt{a^{2}-4 b y}} \\
w & = \pm \frac{2 w_{1}^{\frac{1}{2}} w_{2}^{\frac{1}{2}} a}{b \sqrt{a^{2}-4 b y}}-\frac{2 w_{1}^{\frac{1}{2}} w_{2}^{\frac{1}{2}}}{b},
\end{aligned}
$$

which is the result we've already obtained in (43).

\section{Conclusion}

In this paper we applied principles of duality in a monopolistic case where the single producer has an influence on the price which he charges for the product. We started with the primal approach which includes derivation of the profit function by starting from the production function which describes monopolist's technology. To make the structure of the profit maximization problem in monopolistic case similar to the structure of the profit maximization problem in the perfectly competitive case, the pseudoproduction function is defined. An alternative approach includes derivation of the profit function by starting from the cost function and defined pseudocost function. The implication of duality in microeconomic theory is that all starting functions can be recovered back from derived functions, and the pseudoproduction function and the pseudocost function are recovered from the profit function.

The most important result from an empirical point of view is Hotelling' lemma, whose version is given in the article, and which brings us indirectly from the profit function to the supply function. The limitation of the result is that the demand function has to be known to get us from the pseudoproduction function to the supply function for the given demand of a monopolist. Finally, since the basis for the application of duality in microeconomics is the optimization hypothesis and since it is in reality not always satisfied, the validity of the primal and dual approach in economics can be questioned.

\section{References}

1. Appelbaum, E. (1975). Essays in the Theory and Applications of Duality in Economics, PhD Thesis, Vancouver:University of British Columbia.

2. Blume, L. E. (2008), "Convex Programming", available at http://www.dictionaryofeconomics.com/article?id=pde2008_C000348>

3. Blume, L. E. (2008), "Duality", available at http://www.dictionaryofeconomics.com/article?id=pde2008_D000196>

4. Briec, W, Kerstens, K, Eeckaut, P.V. (2004), "Non-convex Technologies and Cost Functions: Definitions, Duality and Nonparametric Tests of Convexity", Journal of Economics, Vol. 81, pp. 155-192. 
5. Diewert, W.E. (1982), "Duality Approaches to Microeconomic Theory", in Intriligator, M.D, Arrow, K.J. (Eds.), Handbook of Mathematical Economics, Amsterdam: North Holland, pp. 535-599.

6. Kuosmanen, T. (2003), "Duality Theory of Non-convex Technologies", Journal of Productivity Analysis, Vol. 20, pp. 273-304.

7. Lau, L. J. (1978), "Applications of Profit Function", in Fuss, M, McFadden, D. (Eds.), Production Economics: A Dual Approach to Theory and Applications, Vol II, Part IV, Amsterdam: North-Holland, pp. 133-216.

8. Mas - Colell, A, Whinston, M. D, Green, J. (1995). Microeconomic Theory, New York: Oxford University Press.

9. Oraić, M. (2011). Dualnost u mikroekonomskoj teoriji. PhD Thesis. Zagreb: Sveučilište u Zagrebu, Ekonomski fakultet-Zagreb.

10. Shepard, R.W. (1970). Theory of Cost and Production Functions. Princeton: Princeton University Press.

11. Taylor, R.C. (1989). Duality, Optimization, And Microeconomic Theory: Pitfalls for the Applied Researcher. Western Journal of Agricultural Economics, 14: 200-212.

\section{About the authors}

Ilko Vrankić, PhD, is an Associate Professor of Microeconomics and Advanced Microeconomics at the Department of Economic Theory, Faculty of Economics and Business, University of Zagreb. His current research areas are mathematical modelling of economic phenomena and dual approaches to the optimization problems in microeconomic theory. He is the (co) author of number of articles in international and national journals. Author can be contacted at ivrankic@efzg.hr

Mira Krpan, PhD, is an Assistant Professor of Microeconomics and Principles of Economics at the Department of Economic Theory, Faculty of Economics and Business, University of Zagreb. Her current research areas are mathematical modelling of economic phenomena and dual approaches to the optimization problems in microeconomic theory. She is the (co) author of number of articles in international and national journals. Author can be contacted at mira.krpan@efzg.hr 